\title{
Efficacy of intra-articular dexmedetomidine for postoperative analgesia in arthroscopic knee surgery
}

\author{
S Paul ${ }^{1}$, D P Bhattacharjee ${ }^{2}$, S Ghosh ${ }^{3}$, S Dawn ${ }^{2}$, N Chatterjee $^{4}$ \\ (Index words: dexmedetomidine, ropivacaine, arthroscopic knee surgery, intra-articular injection)
}

\begin{abstract}
Introduction Various drugs are administered intraarticularly to provide postoperative analgesia after arthroscopic knee surgery, of which opioids and alpha ${ }_{2}$ agonists could be of particular interest.

Methods Sixty patients undergoing elective knee arthroscopy were randomly assigned to two groups $(\mathrm{n}=30)$. Group $\mathrm{R}$ received $19 \mathrm{ml}$ of $0.25 \%$ ropivacaine and $1 \mathrm{ml}$ of isotonic saline (total volume $20 \mathrm{ml}$ ) intraarticularly. Group RD received $100 \mu \mathrm{g}(1 \mathrm{ml})$ of dexmedetomidine added to $19 \mathrm{ml}$ of $0.25 \%$ ropivacaine intra-articularly (total volume $20 \mathrm{ml}$ ). Analgesic effect was evaluated by measuring pain intensity (VAS score) and duration of analgesia.
\end{abstract}

Results A longer delay was observed between intraarticular injection of study medication and first requirement of supplementary analgesic in group $R D$ (10.84 \pm 2.6 hours) compared to group $R$ (5.38 \pm 1.4 hours). Total consumption of fentanyl citrate in postoperative period was significantly less in group RD. No significant side effects were noted.

Conclusion Dexmedetomidine, added as adjunct to ropivacaine in patients undergoing arthroscopic knee surgery, improve the quality and duration of postoperative analgesia.

Ceylon Medical Journal 2010; 55: 111-15.

\section{Introduction}

Arthroscopic surgery is associated with variable amount of postoperative pain and sometimes the pain is considerable. Postoperative pain relief is essential for early rehabilitation after arthroscopic knee surgery. There has always been a search for a simple method for providing postoperative analgesia in these patients. Intra-articular administration of various drugs provides satisfactory analgesia in immediate postoperative period. Local anaesthetics like lidocaine and bupivacaine, opioids like morphine, alpha-2 adrenoceptor agonists like clonidine and magnesium sulphate have all been tried intraarticularly alone or in combination to provide effective postoperative analgesia [1-5].

Dexmedetomidine is a highly selective alpha-2 adrenergic agonist with sedative, anxiolytic, analgesic, sympatholytic and antihypertensive effects [6]. Activation

Departments of ${ }^{1}$ Pharmacology, ${ }^{2}$ Anaesthesiology and ${ }^{3}$ Orthopaedics, National Medical College, Kolkata, India and ${ }^{4}$ Department of Anaesthesiology, Calcutta Medical Research Institute, Kolkata, India.

Correspondence: SP, e-mail <drsuhritapaul@yahoo.co.in>. Received 8 July and revised version accepted 17 September 2010. Competing interests: none declared. 
of receptors in the brain and spinal cord level inhibits neuronal firing, thereby causing hypotension, bradycardia, sedation and analgesia [7]. Dexmedetomidine has been used intravenously before initiation of regional anaesthesia and it has shown to provide some analgesic effect after arthroscopic knee surgery but there were some adverse haemodynamic effects [8]. Intra-articular administration of dexmedetomidine may be useful to avoid the adverse haemodynamic effects of intravenous administration while still providing the postoperative analgesia.

This placebo controlled, double blind, prospective study is designed to assess and compare the postoperative analgesic effects of intra-articular dexmedetomidine administered as adjuvant with local anaesthetic ropivacaine in patients undergoing arthroscopic knee surgery.

\section{Methods}

The study protocol was approved by the Institutional Ethics Committee of Calcutta National Medical College, Kolkata and informed consent was obtained from every patient in their own language. The sample size was determined by power analysis as follows:

The primary outcome of interest is dichotomous (success/failure, yes/no, etc). For example, $25 \%$ of the participants on the standard therapy had a successful outcome and it is of clinical relevance only if we observe a $40 \%$ (effect size) absolute improvement for those on the study therapy (i.e. $65 \%$ of the participants will have a successful outcome). To determine the number of participants in order to observe a significant difference for a two-sided test of $5 \%$, a simple formula to calculate the sample size is given by $\mathrm{m}$ (size per group) $=\mathrm{cX} \pi_{1}\left(1-\pi_{1}\right)+\pi_{2}\left(1-\pi_{2}\right) /\left(\pi_{1}-\pi_{2}\right)^{2}$. Where, $\mathrm{c}=7.9$ for $80 \%$ power and 10.5 for $90 \%$ power, $\pi_{1}$ and $\pi_{2}$ are the proportion estimates.

Thus from the above example, $\pi_{1}=0.25$ and $\pi_{2}=$ 0.65 . For a $80 \%$ power, we have $\mathrm{m}$ ( (ize per group) $=7.9 \times$ $[0.25(1-0.25)+0.65(1-0.65)] /(0.25-0.65)^{2}=20.49$. Hence $21 \times 2=42$ participants will be needed. Due to availability of participants, we selected 60 patients for our study divided into two groups.

Sixty ASA I -II patients of either sex, aged 18-65 years, undergoing elective knee arthroscopy were randomly assigned to one of the two groups, containing thirty patients each. Surgical procedures consisted of meniscetomies and ligament repair. Patients having history of cardiovascular, cerebrovascular, and respiratory diseases, pregnancy, receiving chronic pain treatment or hypertension treated with $\alpha$ methyldopa, clonidine or $\beta$ adrenergic blockers were excluded from the study. On preoperative rounds, the procedure was explained to patients and they were also taught to interpret the visual analogue scale (VAS) (graded from $0=$ no pain to $10=$ maximum pain).
All patients were kept fasting after midnight and received midazolam $7.5 \mathrm{mg}$ orally as premedication. On the operation table, routine monitoring (ECG, pulse oximetry, non invasive blood pressure) were started and baseline vital parameters like heart rate (HR), blood pressure (systolic, diastolic and mean) and arterial oxygen saturation $\left(\mathrm{SpO}_{2}\right)$ were recorded. An intravenous line was secured.

After preoxygenation for 3 minutes, induction of anaesthesia was done by fentanyl $2 \mu \mathrm{g} / \mathrm{kg}$ and propofol $2 \mathrm{mg} / \mathrm{kg}$. Patients were intubated with appropriate size endotracheal tube after muscle relaxation with vecuronium bromide in a dose of $0.08 \mathrm{mg} / \mathrm{kg}$. Anaesthesia was maintained with $33 \%$ oxygen in nitrous oxide and $1 \%$ isoflurane. Muscle relaxation was maintained by intermittent bolus doses of vecuronium bromide. The patients were mechanically ventilated to keep $\mathrm{EtCO}_{2}$ between 35-40 mm Hg. At the end of surgical procedure, residual neuromuscular paralysis was adequately reversed using intravenous glycopyrrolate and neostigmine and subsequently extubated. Patients were then randomly allocated (using random table assignment) into two groups to receive one of the following intra-articular solutions (prepared by an individual not involved in the study) which were injected into the knee joint through the cannular sheath after withdrawal of camera, by the orthopaedic surgeon (who was also unaware of the nature of the study drugs) before the arthroscope was removed.

In group $\mathrm{R}, 19 \mathrm{ml}$ of $0.25 \%$ ropivacaine and $1 \mathrm{ml}$ isotonic saline (total volume $20 \mathrm{ml}$ ) was administered into the knee joint. Similarly group RD patients received $100 \mu \mathrm{g}$ dexmedetomidine $(1 \mathrm{ml})$ added to $19 \mathrm{ml} 0.25 \%$ ropivacaine (again making a volume of $20 \mathrm{ml}$ ).

All patients were observed postoperatively by resident doctors who were unaware of the study drug. Patients were transferred to postanaesthesia care unit and intensity of pain and vital parameters were assessed after thirty minutes and then hourly for 24 hours. When pain scores (VAS) were 4 or more, patient controlled analgesia (PCA) pump was started to deliver boluses $(25 \mu \mathrm{g})$ of fentanyl citrate as per the requirement of the patient and the time was recorded as the duration of analgesia. Total requirement of fentanyl citrate in 24 hours was also recorded. The patients were monitored for nausea and vomiting, drowsiness, hypotension (defined as systolic blood pressure $>20 \%$ decrease from baseline) and bradycardia (heart rate $<60$ beats/min) during this period.

Data were analysed using computer statistical software system Graph Pad Instat Version 3.05 (Graph Pad software, San Diego, CA) and are presented in a tabulated manner. The results were expressed in mean (SD). Comparisons between groups were performed with the Kruskal Wallis one way ANOVA by ranks or Fisher's exact test for small sample with a 5\% risk. Mann-WhitneyWilcoxon tests were performed when normality tests failed. 


\section{Results}

The two groups were comparable with regard to age, sex, body weight, duration of surgery and type of surgical procedures (Table 1 and 2).

Table1. Patient characteristics of both groups $(n=30)$

\begin{tabular}{lccc}
\hline Variables/Groups & Group $R(n=30)$ & Group $R D(n=30)$ & $p$ value \\
\hline Age (years) & $39.8 \pm 12.6$ & $41.4 \pm 12.8$ & 0.49 \\
Sex (M/F) & $22 / 8$ & $21 / 9$ & \\
Weight $(\mathrm{kg})$ & $54.35 \pm 8.86$ & $56.32 \pm 11.48$ & 0.57 \\
Duration of surgery (mins) & $77.56 \pm 16.4$ & $80.44 \pm 16.76$ & 0.66 \\
\hline
\end{tabular}

The two groups were comparable with regard to types of surgical procedures (Table 2).

Table 2. Types of surgical procedure undergone by the two groups $(n=30)$

\begin{tabular}{lcc}
\hline Surgical procedure & Group $R$ & Group $R D$ \\
\hline Lateral meniscetomy & 4 & 5 \\
Medial meniscetomy & 5 & 6 \\
Medial collateral ligament repair & 4 & 3 \\
Lateral collateral ligament repair & 6 & 5 \\
Anterior cruciate ligament repair & 8 & 7 \\
Posterior cruciate ligament repair & 3 & 4 \\
\hline
\end{tabular}

Intensity of pain was significantly less in group RD compared to group R at 1 hour $(p<0.01), 2$ hours $(p<0.05)$ and 6 hours $(p<0.05)$ following surgery. However, after 10 hours, intensity of pain was comparable in both groups (Table 3 ).

Table 3. Intensity of pain in postoperative period (VAS) (mean \pm SD)

\begin{tabular}{lcc}
\hline Post-operative period & Group $R$ & Group $R D$ \\
\hline 1 hour & $2.8 \pm 0.64$ & $1.9 \pm 0.62^{* *}$ \\
2 hours & $3.01 \pm 0.76$ & $2.3 \pm 0.61^{*}$ \\
6 hours & $4.01 \pm 1.13$ & $3.23 \pm 0.98^{*}$ \\
10 hours & $3.63 \pm 1.02$ & $3.74 \pm 1.02$ \\
14 hours & $3.36 \pm 0.96$ & $3.26 \pm 1.08$ \\
18 hours & $3.5 \pm 1.01$ & $3.32 \pm 1.04$ \\
\hline$* p<0.05 \quad * * p<0.01$ & &
\end{tabular}

The mean duration of analgesia (delay between the intra-articular injection and the first postoperative analgesic demand) was longer in group $\mathrm{RD}$ compared to group $\mathrm{R}(10.84 \pm 2.6$ hour vs. $5.38 \pm 1.4$ hour; $p<0.001)$. Fentanyl consumption in first 24 hours was significantly less in group RD compared to group $\mathrm{R}(p<0.01)$ (Table 4$)$. 
Table 4. Duration of analgesia and opioid consumption in the postoperative period (mean \pm SD)

\begin{tabular}{lccc}
\hline & Group $R$ & Group RD & p value \\
\hline Duration of analgesia (hours) & $5.38 \pm 1.4$ & $10.84 \pm 2.6 * *$ & 0.0001 \\
Fentanyl consumption in 24 hours (mg) & $282.8 \pm 40.12$ & $204.64 \pm 36.48^{*}$ & 0.001 \\
\hline
\end{tabular}

( $p$ value was determined by comparing ropivacaine $(\mathrm{R})$ and dexmedetomidine $(\mathrm{RD})$ groups.)

* Significant difference within groups $* p<0.01 ; * * p<0.001$

Regarding adverse effects, two patients in each group experienced nausea and vomiting. One patient from group RD developed hypotension. Another patient from group RD developed bradycardia. No patients of any group experienced drowsiness. There was no significant difference among the incidence of adverse effects in two groups.

\section{Discussion}

In an attempt to improve the recovery and early rehabilitation after arthroscopic knee surgery, research has been directed towards developing newer techniques for postoperative analgesia. Our study demonstrates a significant increase in postoperative analgesia with dexmedetomidine used with ropivacaine in comparison to ropivacaine alone.

Alpha-2 adrenergic agonists produce their analgesic effects through supraspinal, spinal and peripheral actions [9]. The analgesic effect of intra-articular dexmedetomidine appears to be mainly due to direct local action. However, a central analgesic effect resulting from systemic absorption cannot be excluded. The mechanism of analgesic effect of intra-articular dexmedetomidine might be similar to that of intra-articular clonidine. Clonidine produces analgesia mainly through inhibition of the transmission of nociceptive stimulation in the dorsal horn of spinal cord [10]. Clonidine is reported to mimic the effect of noradrenaline release by descending inhibitory control pathways [11]. Topical administration of clonidine may reduce pain intensity in patients with sympathetically maintained pain, suggesting a peripheral site of action [12]. Dexmedetomidine, like clonidine, may provide local anaesthetic effects which inhibit the conduction of nerve signals through $\mathrm{C}$ and $\mathrm{A} \delta$ fibres and may stimulate the release of enkephalin-like substances at peripheral sites $[13,14]$.

A study evaluating the systemic effects of dexmedetomidine on postoperative analgesia in arthroscopic knee surgery found that buccal dexmedetomidine is better than intramuscular dexmedetomidine in attenuating postoperative pain and reducing diclofenac requirement [15]. In another study dexmedetomidine was used intravenously before epidural anaesthesia [8]. Though dexmedetomidine provided some amount of postoperative analgesia, there were adverse haemodynamic effects like bradycardia and hypotension.

The delay between intra-articular injection of ropivacaine with dexmedetomidine and supplementary analgesic administration by PCA pump was $10.84 \pm 2.6$ hours in our study. When we compare our results with other commonly used intra-articular drugs $[16,17]$ dexmedetomidine and ropivacaine together provide more prolonged analgesia than most other intra-articular agents.

In conclusion dexmedetomidine administered as adjuvant to local anaesthetic ropivacaine improves the quality and duration of postoperative analgesia and reduces the consumption of fentanyl citrate.

\section{Acknowledgements}

Arthroscope was provided by the Orthopaedics Operation Theatre of Calcutta National Medical College and Hospital. Anaesthetic drugs were provided by the Central Store of Calcutta National Medical College and Hospital. Rest of the funding was provided by the investigators themselves.

\section{References}

1. Dahl MR, Dasta JF, Zuelzer W, McSweeney TD. Lidocaine local anaesthesia for arthroscopic knee surgery. Anaesthesia Analgesia 1990; 71: 670-4.

2. Chirwaa SS, MacLeod BA, Day B. Intra-articular bupivacaine after arthroscopic meniscectomy: a randomised double blind controlled study. Arthroscopy 1989; 5: 33-5.

3. Stein C, Comisel K, Haimerl E, Yassouridis A. Analgesic effect of intra-articular morphine after arthroscopic knee surgery. New England Journal of Medicine 1991; 325: 1123-6.

4. Gentili M, Juhel A, Bonnet F. Peripheral analgesic effect of intra-articular clonidine. Pain 1996; 64: 593-6.

5. Bondok RS, Abd El-Hady AM. Intra-articular magnesium is effective for postoperative analgesia in arthroscopic knee surgery. British Journal of Anaesthesia 2006; 97: 389-92.

6. Gerlach AT, Dasta JF. Dexmedetomidine: an updated review. Annals of Pharmacotherapy 2007; 41: 245-52.

7. Hayashi Y, Maze M. Alpha ${ }_{2}$ adrenoceptor agonist and anaesthesia. British Journal of Anaesthesia 1993; 71: 108-18. 
8. Gomez-Vazquez ME, Harnandez-Salazar E, HarnandezJimenez A, et al. Clinical analgesic efficacy and side effects of dexmedetomidine in the early postoperative period after arthroscopic knee surgery. Journal of Clinical Anaesthesia 2007; 19: 576-82.

9. Ebert TJ, Hall JE, Barney JA, Ulrich TD, Colinco MD. The effects of increasing plasma concentrations of dexmedetomidine in humans. Anaesthesiology 2000; 93: 382-94.

10. Yaksh TL, Reddy SVR. Studies in the primate on analgesic effects associated with intrathecal actions of opiates, alpha adrenergic agonists and baclofen. Anaesthesiology 1981; 54: 451-67.

11. Yeomans DC, Clark FM, Paice JA, Proudfit HK. Antinociception induced by electrical stimulation of spinally projecting noradrenergic neurons in the A7 catecholamine cell group of the rat. Brain 1992; 48: 449-61.

12. Davis KD, Treede RD, Raja SN, Meyer RA, Campbell JN. Topical application of clonidine relieves hyperalgesia in patients with sympathetically maintained pain. Pain 1991; 47: 309-17.
13. Butterworth JFV, Strichartz GR. The alpha ${ }_{2}$ adrenergic agonists clonidine and gunafacine produce tonic and phasic block of conduction in rat sciatic nerve fibres. Anaesthesia and Analgesia 1993; 76: 295-301.

14. Nakamura M, Ferreira SH. Peripheral analgesic action of clonidine: mediation by release of endogenous enkephalin like substances. European Journal of Pharmacology 1988; 146: $223-8$

15. Karaaslan D, Peker TT, Alaca A, et al. Comparison of buccal and intramuscular dexmedetomidine premedication for arthroscopic knee surgery. Journal of Clinical Anaesthesia 2006; 18: 589-93.

16. Joshi W, Reuben SS, Kilaru PR, Sklar J, Maciolek H. Postoperative analgesia for outpatient arthroscopic knee surgery with intra-articular clonidine and/or morphine. Anaesthesia and Analgesia 2000; 90: 1102-6.

17. Dal D, Tetic O, Al tunkaya H, Doral MN. The efficacy of intra-articular ketamine for postoperative analgesia in outpatient arthroscopic surgery. Arthroscopy 2004; 20: $300-5$. 